\title{
Maximum Likelihood Estimation of Parameters for Poisson-exponential Distribution Under Progressive Type I Interval Censoring
}

\author{
Peter Tumwa Situma*, Leo Odongo \\ Department of Mathematics and Actuarial Science, Kenyatta University (KU), Nairobi, Kenya \\ Email address: \\ ptsituma@gmail.com (P. T. Situma), leo_odongo@yahoo.co.uk(L. Odongo) \\ ${ }^{*}$ Corresponding author
}

To cite this article:

Peter Tumwa Situma, Leo Odongo. Maximum Likelihood Estimation of Parameters for Poisson-exponential Distribution Under Progressive Type I Interval Censoring. American Journal of Theoretical and Applied Statistics. Vol. 9, No. 2, 2020, pp. 14-20.

doi: $10.11648 /$ j.ajtas.20200902.11

Received: March 13, 2020; Accepted: April 10, 2020; Published: April 23, 2020

\begin{abstract}
This paper considers the problem of estimating the parameters of Poisson-Exponential (PE) distribution under progressive type-I interval censoring scheme. PE is a two-parameter lifetime distribution having an increasing hazard function. It has been applied in complementary risks problems in latent risks, that is in scenarios where maximum lifetime values are observed but information concerning factors accounting for component failure is unavailable. Under progressive type-I interval censoring, observations are known within two consecutively pre-arranged times and items would be withdrawn at pre-scheduled time points. This scheme is most suitable in those cases where continuous examination is impossible. Maximum likelihood estimates of Poisson-Exponential parameters are obtained via Expectation-Maximization (EM) algorithm. The EM algorithm is preferred as it has been confirmed to be a more superior tool when dealing with incomplete data sets having missing values, or models having truncated distributions. Asymptotic properties of the estimates are studied through simulation and compared based on bias and the mean squared error under different censoring schemes and parameter values. It is concluded that for an increasing sample size, the estimated values of the parameters tend to the true value. Among the four censoring schemes considered, the third scheme provides the most precise and accurate results followed by fourth scheme, first scheme and finally the second scheme.
\end{abstract}

Keywords: Poisson-exponential, Progressive Type I Interval Censoring, Maximum Likelihood Estimation, EM Algorithm

\section{Introduction}

In reliability and life testing studies exponential distribution has proved to be a distribution with a simple, elegant and closed form of solution, Tomazella [1]. However, its usefulness is limited based on the fact that it has a constant hazard function. In order to overcome this drawback, different authors have come up with new lifetime distributions based on modification of Exponential distribution. Gupta and Kundu [2] suggested a Generalized Exponential distribution (GED) that fit the data with decreasing and increasing hazard function. Modifying an Exponential distribution to a distribution with a decreasing hazard function was done by Kus [3]. The distribution proposed by Kus [3] was further modified by the inclusion of a power parameter by Barreto and Cribari [4].

Poisson-Exponential (PE) distribution is a two-parameter lifetime distribution which was first introduced by Cancho et al. [5]. Its failure rate increases with time. The distribution is widely applicable in complementary risk (CR) studies. Louzada-Neto et al. [6] studied statistical properties of PE distribution and discussed Bayes estimators based on Squared Error Loss Function (SELF). Singh et al. [7] obtained the Maximum Likelihood Estimators and Bayes estimators of the parameters of the PE distribution under symmetric and asymmetric loss function and compared the proposed estimators in terms of their risks with the Maximum Likelihood Estimators. Rodrigues et al. [8] considered different estimation methods for parameters of PE distribution. Gitahi et al. [9] obtained the Maximum Likelihood Estimators of the parameters of PE distribution 
based on progressively type II censoring via the EM algorithm. Belaghi et al. [10] considered prediction and estimation of lifetime data following PE distribution under type II censoring.

In this study, we assume that lifetimes have PE distribution. This distribution has been applied in complementary risk problems in latent risks, that is in scenarios where maximum lifetime values are observed but information concerning factors accounting for component failure is unavailable.

A random variable $\mathrm{X}$ is said to have a Poisson-Exponential distribution if its probability density function (PDF) and cumulative distribution function (CDF) are respectively given by

$$
\begin{gathered}
f(x ; \theta, \lambda)=\frac{\theta \lambda e^{-\lambda x-\theta e^{-\lambda x}}}{1-e^{-\theta}}, x>0, \theta>0, \lambda>0 \\
F(x ; \theta, \lambda)=1-\frac{1-e^{-\theta e^{-\lambda x}}}{1-e^{-\theta}}, x>0, \theta>0, \lambda>0
\end{gathered}
$$

Where $\theta>0$ and $\lambda>0$ are respectively, the shape and scale parameters of the distribution. Louzada-Neto et al. [6] noted that the parameters $\lambda$ and $\theta$ can be directly interpreted in terms of CR. That is; $\lambda$ represents lifetime failure rate while $\theta$ denotes the mean number of CR.

In lifetime analysis, censoring occurs when lifetime of an item is not observed. Various types of censoring exist of which Type I and type II censoring schemes are the most common. The time $\mathrm{T}$ of termination of the experiment is pre-arranged in type I censoring. On the other hand, the experiment continues until a pre-arranged number of failures occur in type II censoring. However, the two schemes do not permit removal of experimental units at any other point other than the final termination point of the experiment. In many practical situations, there is need for the removal of test items at different points prior to the termination of the experiment. Progressive censoring schemes allow the removal of experimental units at different time points other than the termination point of the experiment as discussed in Cohen [11]. For detailed discussion on progressive type I and type II censoring schemes, the reader may refer to Balakrishnan [12] and Balakrishnan and Cramer [13].

Aggarwala [14] proposed progressive type I (PTI) interval censoring and provided the statistical inference for the exponential distribution. Under PTI interval censoring, observations are known within two consecutively pre-scheduled times and items would be withdrawn at pre-scheduled time points. $\mathrm{Ng}$ and Wang [15] dealt with the estimation of Weibull distribution parameters basing on the PTI interval-censored sample. Cheng et al. [16] introduced a new algorithm for maximum likelihood estimation under PTI interval-censored data. Chen and Lio [17] estimated parameters of Generalized exponential distribution under PTI interval censoring. Lio et al. [18] considered estimation of parameters of Generalized Rayleigh distribution based on progressively type I interval-censored data. Lin and Lio [19] estimated the parameters of Weibull and Generalized exponential distribution under PTI interval censoring by Bayesian method. Teimouri and Gupta [20] considered estimation methods for the Gompertz-makeham distribution under PTI interval censoring. Recently, Singh and Tripathi [21] estimated Inverse Weibull distribution parameters under PTI interval censoring in classical and Bayesian frameworks.

In this paper, we consider Maximum Likelihood Estimation of parameters of PE distribution based on the PTI interval censoring scheme. We propose to use EM algorithm to compute the MLEs. We also assess the precision and accuracy of the MLEs of the parameters of PE distribution under different censoring schemes and parameter values using simulation studies.

The rest of this paper is organized as follows: In section 2, we briefly describe progressive type I interval censoring scheme. In addition, we obtain Maximum likelihood estimates of PE distribution based on PTI interval censoring via EM algorithm. In section 3, we conduct simulation study. Finally, the conclusions are given in section 4.

\section{Parameter Estimation}

\subsection{Progressive Type I Interval Censoring Scheme}

On a lifetime experiment, let $n$ units be placed on a life testing simultaneously at time $t_{0}=0$ and under inspection at $m$ pre-arranged times $t_{1}<t_{2}<\ldots<t_{m}$. Let $t_{m}$ be the planned time for test termination when all surviving items are removed. The number of failures, $X_{i}$, and the number of randomly removed surviving items from the test, $R_{i}$, is recorded in the interval $\left(t_{i-1}, t_{i}\right]$ at time $t_{i}$ where $i=1,2, \ldots, m$. In the interval $\left(t_{i-1}, t_{i}\right]$, the number of surviving items, $Y_{i}$, is a random variable and $R_{i} \leq Y_{i}$. At time $t_{i}, R_{i}$ could be determined through pre-determined percentages of surviving remaining items. Given pre-determined percentages $p_{1}, p_{2}, \ldots, p_{m-1}$ and $p_{m}=1$ for removal at time $t_{1}<t_{2}<\ldots<t_{m}$ respectively, $R_{i}=p_{i} Y_{i}$. Hence, the PTI interval-censored sample is represented by $\left\{X_{i}, R_{i}, t_{i}\right\}$.

\subsection{Maximum Likelihood Estimation Based on Progressive Type I Interval Censoring Scheme}

Given a PTI interval-censored sample, $\left\{X_{i}, R_{i}, t_{i}\right\}$ for $i=1,2, \ldots, m$ of size $n$, from a continuous lifetime distribution with $(\mathrm{CDF}), F(t ; \Theta)$, where parameter vector $\Theta=(\theta, \lambda)$, the likelihood function is derived as follows, according to Aggarwala [14].

$$
\begin{aligned}
& L(\Theta) \alpha\left[F\left(t_{1} ; \Theta\right)\right]^{X_{1}}\left[1-F\left(t_{1} ; \Theta\right)\right]^{R_{1}} \times\left[F\left(t_{2} ; \Theta\right)-F\left(t_{1} ; \Theta\right)\right]^{X_{2}}\left[1-F\left(t_{2} ; \Theta\right)\right]^{R_{2}} \\
& \times \ldots \times\left[F\left(t_{m} ; \Theta\right)-F\left(t_{m-1} ; \Theta\right)\right]^{X_{m}}\left[1-F\left(t_{m} ; \Theta\right)\right]^{R_{m}}
\end{aligned}
$$




$$
L(\Theta) \alpha \prod_{i=1}^{m}\left[F\left(t_{i} ; \Theta\right)-F\left(t_{i-1} ; \Theta\right)\right]^{X_{i}}\left[1-F\left(t_{i} ; \Theta\right)\right]^{R_{i}}
$$

The likelihood function (3) reduces to the likelihood function for the conventional type I censored case if $R_{1}=R_{2}=\ldots=R_{m-1}=0$

By substituting (2) in (3) and simplifying, we obtain

$$
l(\Theta)=\sum_{i=1}^{m} X_{i} \log \left[e^{-\theta e^{-\lambda t_{i}}}-e^{-\theta e^{-\lambda_{i-1}}}\right]+\sum_{i=1}^{m} R_{i} \log \left[1-e^{-\theta e^{-\lambda_{i}}}\right]-\left(\sum_{i=1}^{m} X_{i}+\sum_{i=1}^{m} R_{i}\right) \log \left[1-e^{-\theta}\right]
$$

Differentiating (5) partially with respect to $\theta$ and $\lambda$ and equating to zero, we obtain the following normal equations

$$
\begin{gathered}
\frac{\partial l}{\partial \theta}=\frac{\sum_{i=1}^{m} X_{i} e^{-\lambda t_{i-1}} e^{-\theta e^{-\lambda i_{i-1}}}-\sum_{i=1}^{m} X_{i} e^{-\lambda t_{i}} e^{-\theta e^{-\lambda i_{i}}}}{e^{-\theta e^{-\lambda \lambda_{i}}}-e^{-\theta e^{-\lambda \lambda_{i-1}}}}+\frac{\sum_{i=1}^{m} R_{i} e^{-\lambda t_{i}} e^{-\theta e^{-\lambda \lambda_{i}}}}{1-e^{-\theta e^{-\lambda \lambda_{i}}}}-\frac{\left(\sum_{i=1}^{m} X_{i}+\sum_{i=1}^{m} R_{i}\right) e^{-\theta}}{1-e^{-\theta}}=0 \\
\frac{\partial l}{\partial \lambda}=\frac{\theta \sum_{i=1}^{m} X_{i} t_{i} e^{-\lambda t_{i}} e^{-\theta e^{-\lambda t_{i}}}-\theta \sum_{i=1}^{m} X_{i} t_{i-1} e^{-\lambda t_{i-1}} e^{-\theta e^{-\lambda t_{i-1}}}}{e^{-\theta e^{-\lambda \lambda_{i}}}-e^{-\theta e^{-\lambda t_{i-1}}}}-\frac{\theta \sum_{i=1}^{m} R_{i} t_{i} e^{-\lambda t_{i}} e^{-\theta e^{-\lambda_{i}}}}{1-e^{-\theta e^{-\lambda i_{i}}}}=0
\end{gathered}
$$

Clearly, normal equations (6) and (7) cannot yield solution to $\theta$ and $\lambda$ in a closed-form. We therefore introduce the Expectation-Maximization (EM) algorithm to obtain the MLEs of $\theta$ and $\lambda$.

\subsection{EM Algorithm}

The EM Algorithm was introduced by Dempster et al. [22] to deal with incomplete data problems. PTI interval censoring can be considered as an incomplete data problem and therefore EM Algorithm is used as the most suitable method in obtaining the MLEs of the unknown parameters. $E M$ is an iterative procedure whereby each iteration consists of two steps that is the Expectation step (E-step) and the Maximization step (M-step).

Let $X_{i}$ represent the number of failed units observed in the interval $\left(t_{i-1}, t_{i}\right]$ and $R_{i}$ represent withdrawn units from the experiment at a specified time $t_{i}$ where $i=1,2, \ldots, m$. Additionally, we assume $T_{i j}$ and $Z_{i j}$ to represent lifetimes of observed failure for $j=1,2, \ldots, X_{i}$ and lifetimes of the missing units for $j=1,2, \ldots, R_{i}$ respectively from an experiment at time $t_{i}$ where $i=1,2, \ldots, m$. Therefore, under PTI interval censoring scheme, the likelihood function of the complete sample is given by

$$
L(\Theta) \alpha \prod_{i=1}^{m}\left[\prod_{j=1}^{X_{i}} f\left(T_{i j} ; \Theta\right) \prod_{j=1}^{R_{i}} f\left(Z_{i j} ; \Theta\right)\right]
$$

Introducing logs on (8) we get

$$
l(\Theta)=\sum_{i=1}^{m} \sum_{j=1}^{X_{i}} \ln f\left(T_{i j} ; \Theta\right)+\sum_{i=1}^{m} \sum_{j=1}^{R_{i}} \ln f\left(Z_{i j} ; \Theta\right)
$$

substituting for $f\left(T_{i j} ; \Theta\right)$ and $f\left(Z_{i j} ; \Theta\right)$ with (1) yields

$$
l(\Theta)=\sum_{i=1}^{m} \sum_{j=1}^{X_{i}} \ln \left[\frac{\theta \lambda e^{-\lambda T_{i j}-\theta e^{-\lambda T_{i j}}}}{1-e^{-\theta}}\right]+\sum_{i=1}^{m} \sum_{j=1}^{R_{i}} \ln \left[\frac{\theta \lambda e^{-\lambda Z_{i j}-\theta e^{-\lambda z_{i j}}}}{1-e^{-\theta}}\right]
$$

Simplification of equation (9) yields

$$
l(\Theta)=n \ln (\theta \lambda)-\lambda \sum_{i=1}^{m} \sum_{j=1}^{X_{i}}\left(T_{i j}\right)-\theta \sum_{i=1}^{m} \sum_{j=1}^{X_{i}}\left(e^{-\lambda T_{i j}}\right)-\lambda \sum_{i=1}^{m} \sum_{j=1}^{R_{i}}\left(Z_{i j}\right)-\theta \sum_{i=1}^{m} \sum_{j=1}^{R_{i}}\left(e^{-\lambda Z_{i j}}\right)-n \ln \left(1-e^{-\theta}\right)
$$

Where $n=\sum_{i=1}^{m}\left(X_{i}+R_{i}\right)$

The lifetimes of the $X_{i}$ failures in the $i^{\text {th }}$ interval $\left(t_{i-1}, t_{i}\right]$ are independent and follow a doubly truncated PE distribution from left and right at $t_{i-1}$ and $t_{i}$ respectively and the lifetimes of the $R_{i}$ censored units in the $i^{t h}$ interval $\left(t_{i-1}, t_{i}\right]$ are independent and follow a truncated PE distribution from left at $t_{i}$ for $i=1,2, \ldots, m$.

The E-step requires the construction of pseudo-log-likelihood function. This is achieved by computing the conditional expectations and then replacing them in the log-likelihood function (10). For our case the required conditional expectations are 


$$
\begin{aligned}
& E_{1 i}=E\left[T_{i j} / T_{i j} \in\left(t_{i-1}, t_{i}\right] ; \Theta\right] \\
& E_{3 i}=E\left[Z_{i j} / Z_{i j} \in\left(t_{i}, \infty\right) ; \Theta\right]
\end{aligned}
$$

Conditional expectations $E_{1 i}, E_{2 i}, E_{3 i}$ and $E_{4 i}$ are computed by application of the general form in (11) in conjunction with some integration techniques. The required expected values of a doubly truncated distribution at $p$ and $q$ from left and right respectively with $0<p<q<\infty$ for EM algorithm is given by

$$
\begin{aligned}
& E_{2 i}=E\left[e^{-\lambda T_{i j}} / T_{i j} \in\left(t_{i-1}, t_{i}\right] ; \Theta\right] \\
& E_{4 i}=E\left[e^{-\lambda Z_{i j}} / Z_{i j} \in\left(t_{i}, \infty\right) ; \Theta\right]
\end{aligned}
$$

$$
E_{\theta, \lambda}[y \mid y \in(p, q]]=\frac{\int_{p}^{q} y f(y ; \theta, \lambda) d y}{F(q ; \theta, \lambda)-F(p ; \theta, \lambda)}
$$

Replacing the conditional expectations $E_{1 i}, E_{2 i}, E_{3 i}$ and $E_{4 i}$ in equation (10) completes the E-step. We get the pseudo-log-likelihood function as

$$
l(\Theta)=n \ln (\theta \lambda)-\lambda \sum_{i=1}^{m} X_{i} E_{1 i}-\theta \sum_{i=1}^{m} X_{i} E_{2 i}-\lambda \sum_{i=1}^{m} R_{i} E_{3 i}-\theta \sum_{i=1}^{m} R_{i} E_{4 i}-n \ln \left(1-e^{-\theta}\right)
$$

After substituting the conditional expectations, partial derivatives of (12) with respect to parameters are derived in order to maximize the pseudo-log-likelihood function as follows.

$$
\frac{\partial l(\Theta)}{\partial \lambda}=\frac{n}{\lambda}-\sum_{i=1}^{m} X_{i} E_{1 i}-\sum_{i=1}^{m} R_{i} E_{3 i}
$$

and

$$
\frac{\partial l(\Theta)}{\partial \theta}=\frac{n}{\theta}-\frac{n}{e^{\theta}-1}-\sum_{i=1}^{m} X_{i} E_{2 i}-\sum_{i=1}^{m} R_{i} E_{4 i}
$$

Suppose that $\left(\theta^{(k)}, \lambda^{(k)}\right)$ are estimates of $(\theta, \lambda)$ at the $k^{\text {th }}$ step. Then, by equating (13) and (14) to zero, we obtain

$$
\frac{n}{\lambda^{(k)}}=\sum_{i=1}^{m} X_{i} E_{1 i}(\theta ; \lambda)+\sum_{i=1}^{m} R_{i} E_{3 i}(\theta ; \lambda)
$$

and

$$
\frac{n}{\theta^{(k)}}=\frac{n}{e^{\theta}-1}+\sum_{i=1}^{m} X_{i} E_{2 i}(\theta ; \lambda)+\sum_{i=1}^{m} R_{i} E_{4 i}(\theta ; \lambda)
$$

The M-step requires solutions to equations (15) and (16) to obtain the next values $\lambda^{(k+1)}$ and $\theta^{(k+1)}$ of $\lambda$ and $\theta$ given respectively by

$$
\lambda^{(k+1)}=\frac{n}{\sum_{i=1}^{m} X_{i} E_{1 i}\left(\theta^{(k)} ; \lambda^{(k)}\right)+\sum_{i=1}^{m} R_{i} E_{3 i}\left(\theta^{(k)} ; \lambda^{(k)}\right)}
$$

and

$$
\theta^{(k+1)}=\frac{n}{\frac{n}{e^{g^{(k)}}-1}+\sum_{i=1}^{m} X_{i} E_{2 i}\left(\theta^{(k)} ; \lambda^{(k)}\right)+\sum_{i=1}^{m} R_{i} E_{4 i}\left(\theta^{(k)} ; \lambda^{(k)}\right)}
$$

Checking convergence, if the convergence occurs at $\left(\lambda^{(k)}, \theta^{(k)}\right)$ then the current $\lambda^{(k)}$ and $\theta^{(k)}$ are the approximated MLEs of $\lambda$ and $\theta$ via EM algorithm. Otherwise set $k=k+1$ and go back to equations (17) and (18). The process is repeated until convergence occurs. At convergence $\left|\theta^{(k+1)}-\theta^{(k)}\right|<\epsilon$ and $\left|\lambda^{(k+1)}-\lambda^{(k)}\right|<\epsilon$ for some given $\in>0$, say $\in=0.0001$.

\section{Simulation Study}

Simulation study was conducted to investigate the behavior of the proposed MLEs of PE distribution parameters under PTI interval censoring scheme via EM algorithm on simulated data. The simulation was conducted in $\mathrm{R}$ language, a software package that was designed by Ihaka and Gentleman [23].

\subsection{Simulation Algorithm}

According to the algorithm proposed in Aggarwala [14], a progressively type I interval-censored data $\left\{X_{l}, R_{l}, t_{l}\right\}$ for $l=1,2, \ldots, m$ from PE distribution with $\mathrm{CDF}$ (2) is generated as follows: Let $X_{0}=0, R_{0}=0$ and $\Theta \in(\theta, \lambda)$.

$$
\begin{aligned}
& X_{l} \mid X_{l-1}, \ldots, X_{0}, R_{1-1}, \ldots, R_{0} \sim \operatorname{rbinom}\left[n-\sum_{k=1}^{l-1}\left(X_{k}+R_{k}\right), \frac{F\left(t_{l} ; \Theta\right)-F\left(t_{l-1} ; \Theta\right)}{1-\sum_{k=1}^{l-1}\left[F\left(t_{k} ; \Theta\right)-F\left(t_{k-1} ; \Theta\right)\right]}\right] \\
& =\operatorname{rbinom}\left[n-\sum_{k=1}^{l-1}\left(X_{k}+R_{k}\right), \frac{F\left(t_{l} ; \Theta\right)-F\left(t_{l-1} ; \Theta\right)}{1-F\left(t_{l-1} ; \Theta\right)}\right]=\operatorname{rbinom}\left[n-\sum_{k=1}^{l-1}\left(X_{k}+R_{k}\right), \frac{e^{-\theta e^{-\lambda l l}}-e^{-\theta e^{-\lambda l_{l-1}}}}{1-e^{-\theta e^{-\lambda l l-1}}}\right]
\end{aligned}
$$




$$
R_{l}=\text { floor }\left\{p_{l} \times\left[n-\sum_{k=1}^{l-1}\left(X_{k}+R_{k}\right)-X_{l}\right]\right\}
$$

Where floor () returns the greatest integer equal to or less than the argument.

Clearly, if $p_{1}=p_{2}=\ldots=p_{m-1}=0 \quad, \quad$ then $R_{1}=R_{2}=\ldots=R_{m-1}=0$ and hence
$X_{1}, X_{2}, \ldots, X_{m}, X_{m+1}=R_{m}$ becomes a simulated sample from the conventional type I interval censoring. The above algorithm is an improvement from the one suggested by Kemp and Kemp [24].

To investigate the behavior of the MLEs, we consider the schemes below, similar to schemes used in Ng and Wang [15], Chen and Lio [17] and Lio et al. [18].

$$
\begin{aligned}
& \text { scheme } 1, p_{(1)}=(0.25,0.25,0.25,0.25,0.5,0.5,0.5,0.5,1) \\
& \text { scheme } 2, p_{(2)}=(0.5,0.5,0.5,0.5,0.25,0.25,0.25,0.25,1) \\
& \text { scheme } 3, p_{(3)}=(0,0,0,0,0,0,0,0,1) \\
& \text { scheme } 4, p_{(4)}=(0.25,0,0,0,0,0,0,0,1)
\end{aligned}
$$

The schemes are chosen to specify the percentage of surviving units to be withdrawn at the 9 censoring and monitoring points. Whereby in $p_{(1)}$ for the first four intervals the removal is lighter as compared to the last four intervals, while in $p_{(2)}$ the reverse scenario of $p_{(1)}$ is applied. In $p_{(3)}$ no removal is done prior to termination which is a case similar to conventional type I interval censoring. Lastly, in $p_{(4)}$ removal is conducted at the left-most and right-most ends.

We considered the parameter values and sample sizes respectively as

\subsection{Simulation Results}

$(\theta, \lambda) \in\{(0.02,0.2),(0.02,0.4),(0.03,0.2),(0.03,0.4)\} \quad$ and $\mathrm{n}=20,200$

In this paper, convergence is assumed to occur when the absolute difference between successive estimates is less than 0.0001 .

Suppose $\hat{\Theta}_{i}$ is the MLE of $\Theta$ for the $i^{\text {th }}$ replication of the simulated EM algorithm, then from the simulation runs the absolute value of bias and mean squared error (MSE) of $\stackrel{\Lambda}{\Theta}$

\begin{tabular}{|c|c|c|c|c|c|c|c|}
\hline \multirow{2}{*}{$\mathbf{n}$} & \multirow{2}{*}{$\begin{array}{l}\text { Censoring } \\
\text { scheme }\end{array}$} & \multicolumn{6}{|c|}{ Estimated values } \\
\hline & & $\hat{\theta}$ & $\hat{\lambda}$ & Bias $\hat{\theta}$ & Bias $\hat{\lambda}$ & M S E $\hat{\theta}$ & $\operatorname{MSE} \hat{\lambda}$ \\
\hline \multirow{5}{*}{20} & 1 & 0.02017589 & 0.1010482 & 0.0001759 & 0.0989518 & $3.094 \mathrm{E}-08$ & 0.00979 \\
\hline & 2 & 0.02022346 & 0.0710573 & 0.0002235 & 0.1289427 & 4.993E-08 & 0.01663 \\
\hline & 3 & 0.02010807 & 0.1428157 & 0.0001081 & 0.0571843 & $1.168 \mathrm{E}-08$ & 0.00327 \\
\hline & 4 & 0.02014845 & 0.1410200 & 0.0001484 & 0.0589800 & $2.204 \mathrm{E}-08$ & 0.00348 \\
\hline & 1 & 0.02015016 & 0.1226911 & 0.0001502 & 0.0773089 & $2.255 \mathrm{E}-08$ & 0.00598 \\
\hline \multirow[t]{3}{*}{200} & 2 & 0.02022257 & 0.0774668 & 0.0002226 & 0.1225332 & 4.954E-08 & 0.01501 \\
\hline & 3 & 0.02006497 & 0.1770925 & 0.0000650 & 0.0229075 & $4.221 \mathrm{E}-09$ & 0.00052 \\
\hline & 4 & 0.02012954 & 0.1453538 & 0.0001295 & 0.0546462 & $1.678 \mathrm{E}-08$ & 0.00299 \\
\hline
\end{tabular}
are respectively computed by applying the formulae below:-

i. $\operatorname{Bias}(\hat{\Theta})=\frac{1}{100} \sum_{i=1}^{100}\left|\Theta-\hat{\Theta}_{i}\right|$, where $\Theta=(\theta, \lambda)$

ii. $\operatorname{MSE}(\stackrel{\Lambda}{\Theta})=\frac{1}{100} \sum_{i=1}^{100}\left(\Theta-\Theta_{i}\right)^{2}$

Table 1. Bias and MSE of $\hat{\theta}$ and $\hat{\lambda}$ under different censoring schemes when $\theta=0.02$ and $\lambda=0.2$.

Table 2. Bias and MSE of $\hat{\theta}$ and $\hat{\lambda}$ under different censoring schemes when $\theta=0.02$ and $\lambda=0.4$.

\begin{tabular}{llllllll}
\hline \multirow{2}{*}{$\mathbf{n}$} & Censoring & \multicolumn{2}{l}{ Estimated values } & & & \\
\cline { 2 - 8 } & scheme & $\hat{\theta}$ & $\hat{\lambda}$ & Bias $\hat{\theta}$ & Bias $\hat{\lambda}$ & M S E $\hat{\theta}$ & M S E $\hat{\lambda}$ \\
\hline \multirow{2}{*}{20} & 1 & 0.02025872 & 0.2181204 & 0.0002587 & 0.1818796 & $6.694 \mathrm{E}-08$ & 0.03308 \\
& 2 & 0.02031863 & 0.1479301 & 0.0003186 & 0.2520699 & $1.015 \mathrm{E}-07$ & 0.06354 \\
& 3 & 0.02013722 & 0.2795695 & 0.0001372 & 0.1204305 & $1.883 \mathrm{E}-08$ & 0.01450 \\
& 4 & 0.02021796 & 0.2531296 & 0.0002180 & 0.1468704 & $4.751 \mathrm{E}-08$ & 0.02157 \\
& 1 & 0.02021691 & 0.2446643 & 0.0002169 & 0.1553357 & $4.705 \mathrm{E}-08$ & 0.02413 \\
& 1 & 0.02027654 & 0.1842143 & 0.0002765 & 0.2157857 & $7.647 \mathrm{E}-08$ & 0.04656 \\
& 2 & 0.02010860 & 0.3091464 & 0.0001086 & 0.0908536 & $1.179 \mathrm{E}-08$ & 0.00825 \\
& 3 & 0.02017807 & 0.2812259 & 0.0001781 & 0.1187741 & $3.171 \mathrm{E}-08$ & 0.01411 \\
\hline
\end{tabular}


Table 3. Bias and MSE of $\hat{\theta}$ and $\hat{\lambda}$ under different censoring schemes when $\theta=0.03$ and $\lambda=0.2$.

\begin{tabular}{|c|c|c|c|c|c|c|c|}
\hline \multirow{2}{*}{$\mathbf{n}$} & \multirow{2}{*}{$\begin{array}{l}\text { Censoring } \\
\text { scheme }\end{array}$} & \multicolumn{6}{|c|}{ Estimated values } \\
\hline & & $\hat{\theta}$ & $\hat{\lambda}$ & Bias $\hat{\theta}$ & Bias $\hat{\lambda}$ & M S E $\hat{\theta}$ & $\mathrm{MSE} \hat{\lambda}$ \\
\hline \multirow{5}{*}{20} & 1 & 0.03038365 & 0.1005903 & 0.0003837 & 0.0994097 & $1.472 \mathrm{E}-07$ & 0.00988 \\
\hline & 2 & 0.03048287 & 0.07007791 & 0.0004829 & 0.12992209 & $2.332 \mathrm{E}-07$ & 0.01688 \\
\hline & 3 & 0.03023907 & 0.1426577 & 0.0002391 & 0.0573423 & $5.715 \mathrm{E}-08$ & 0.00329 \\
\hline & 4 & 0.03032899 & 0.1409852 & 0.0003290 & 0.0590148 & $1.082 \mathrm{E}-07$ & 0.00348 \\
\hline & 1 & 0.03035381 & 0.1162870 & 0.0003538 & 0.0837130 & $1.252 \mathrm{E}-07$ & 0.00701 \\
\hline \multirow[t]{3}{*}{200} & 2 & 0.03048001 & 0.07636214 & 0.0004800 & 0.12363786 & $2.034 \mathrm{E}-07$ & 0.01529 \\
\hline & 3 & 0.03014612 & 0.1766993 & 0.0001461 & 0.0233007 & $2.135 \mathrm{E}-08$ & 0.00054 \\
\hline & 4 & 0.03028618 & 0.1451920 & 0.0002862 & 0.0548080 & $8.190 \mathrm{E}-08$ & 0.00300 \\
\hline
\end{tabular}

Table 4. Bias and MSE of $\hat{\theta}$ and $\hat{\lambda}$ under different censoring schemes when $\theta=0.03$ and $\lambda=0.4$.

\begin{tabular}{|c|c|c|c|c|c|c|c|}
\hline \multirow{2}{*}{$\mathbf{n}$} & \multirow{2}{*}{$\begin{array}{l}\text { Censoring } \\
\text { scheme }\end{array}$} & \multicolumn{6}{|c|}{ Estimated values } \\
\hline & & $\hat{\theta}$ & $\hat{\lambda}$ & Bias $\hat{\theta}$ & Bias $\hat{\lambda}$ & M S E $\hat{\theta}$ & $\operatorname{MSE} \hat{\lambda}$ \\
\hline \multirow{5}{*}{20} & 1 & 0.03061982 & 0.2177433 & 0.0006198 & 0.1822567 & $3.842 \mathrm{E}-07$ & 0.03322 \\
\hline & 2 & 0.03070269 & 0.1469932 & 0.0007027 & 0.2530068 & $4.938 \mathrm{E}-07$ & 0.06401 \\
\hline & 3 & 0.03037520 & 0.2795617 & 0.0003752 & 0.1204383 & $1.408 \mathrm{E}-07$ & 0.01451 \\
\hline & 4 & 0.03048842 & 0.2529547 & 0.0004884 & 0.1470453 & $2.386 \mathrm{E}-07$ & 0.02162 \\
\hline & 1 & 0.03048228 & 0.2442800 & 0.0004823 & 0.1557200 & $2.326 \mathrm{E}-07$ & 0.02425 \\
\hline \multirow[t]{3}{*}{200} & 2 & 0.03061033 & 0.1798701 & 0.0006103 & 0.2201299 & $3.725 \mathrm{E}-07$ & 0.04846 \\
\hline & 3 & 0.03024274 & 0.3091344 & 0.0002427 & 0.0908656 & $5.892 \mathrm{E}-08$ & 0.00826 \\
\hline & 4 & 0.03039780 & 0.2810305 & 0.0003978 & 0.1189695 & $1.582 \mathrm{E}-07$ & 0.01415 \\
\hline
\end{tabular}

A summary of results from Tables 1-4 is provided below:

i. The MSE and bias of the estimates decrease as the sample size increase from $n=20$ to $n=200$ for each censoring scheme. These imply that the estimates become more precise and accurate as the sample size increases.

ii. Among the four censoring schemes, the third scheme $p_{(3)}$ provides the most precise and accurate results as seen from the MSE and bias values, followed by scheme $p_{(4)}, p_{(1)}$ and finally $p_{(2)}$. Similar performance among the four schemes is observed when the sample size is increased from $n=20$ to $n=200$.

The results of the performance comparisons among these four censoring schemes are similar to the results obtained in Aggarwala [14], Ng and Wang [15], Chen and Lio [17] and Lio et al. [18].

These phenomena are expected since the third censoring scheme $p_{(3)}$, could have the largest number of failure items observed before the termination of the life-testing, followed by $p_{(4)}, p_{(1)}$ and finally $p_{(2)}$. Intuitively, these are also consistent with the statistical theory that the larger the "sample size" is the more accurate the parameter estimate is.

iii. As for the performance among the assumed true parameters, it is observed that when the values are varied from $(\theta=0.02$ and $\lambda=0.2) \quad$ to ( $\theta=0.03$ and $\lambda=0.4)$ the estimators become less accurate and less precise as depicted from the bias and MSE values from Table 1 and Table 4.

\section{Conclusion}

The study has addressed the problem of the Maximum Likelihood Estimation of parameters for Poisson-Exponential distribution based on progressive type I interval-censored data. The maximum likelihood estimates were obtained using the EM algorithm.

A comparison of the MLEs obtained was made by simulation under four different censoring schemes and various parameter values. It was observed that:

i. For an increasing sample size, the estimated values of the parameters became closer to the true values.

ii. Among the four censoring schemes considered, the third scheme provided the most accurate and precise results then schemes four, one and lastly scheme two.

\section{References}

[1] Tomazella, V. L. D., Cancho, V. G., and Louzada, F. (2013). Bayesian reference analysis for the Poisson-Exponential lifetime distribution. Chilean Journal of Statistics; 4 (1): 99-113.

[2] Gupta, R. D., and Kundu, D. (1999). Generalized exponential distributions. Australian and New Zealand Journal of Statistics; 41: 73-188.

[3] Kus, C. (2007). A new lifetime distribution. Computational Statistics and Data Analysis; 51: 4497-4509.

[4] Barreto, S. W., and Cribari, N. F. (2009). A generalization of Exponential-Poisson distribution. Statistics and Probability Letters; 79: 2493-2500.

[5] Cancho, V. G., Louzada-Neto, F. and Barriga, G. D. C. (2011). The Poisson-Exponential lifetime distribution. Computational Statistics and Data Analysis; 55: 677-686.

[6] Louzada-Neto, F., Cancho, V. G., and Barriga, G. D. C. (2011). The Poisson-Exponential distribution: a Bayesian approach. Journal of Applied Statistics; 38: 1239-1248. 
[7] Singh S. K., Singh, U. and Manoj, K. M. (2014). Estimation for the Parameter of Poisson-Exponential Distribution under Bayesian Paradigm, Journal of Data Science; 12: 157-173.

[8] Rodrigues, G. C., Louzada, F., and Ramos, P. L. (2016). Poisson-exponential distribution: different methods of estimation. Journal of Applied Statistics; 45 (1): 128-144.

[9] Gitahi, J. N., Kung'u, J., and Odongo, L. (2017). Estimation of the Parameters of Poisson-Exponential Distribution Based on Progressively Type II censoring using the Expectation-Maximization Algorithm. American Journal of Theoretical and Applied Statistics; 6: 141-149.

[10] Belaghi, R. A., Asl, M. N., Alma, O. G., Singh, S., and Vasfi, M. (2018). Estimation and Prediction for the Poisson-Exponential Distribution Based on Type-II Censored Data. American Journal of Mathematical and Management Sciences. DOI: 10.1080/01966324.2018.1484827.

[11] Cohen, A. C. (1963). Progressively censored samples in life testing. Technometrics; 5: 327-339.

[12] Balakrishnan, N. (2007). Progressive censoring methodology: an appraisal. Research Papers in Economics; 16: 211-259.

[13] Balakrishnan, N., and Cramer, E. (2014). The art of Progressive censoring: application to Reliability and Quality. Basel: Birkhauser.

[14] Aggarwala, R. (2001). Progressive interval censoring: some mathematical results with applications to inference. Communication in Statistics Theory and Methods; 30: 1921-1935.

[15] Ng, H. K. T., and Wang, Z. (2009). Statistical estimation for the parameters of Weibull distribution based on progressively type-I interval-censored sample. Journal of Statistical Computation and Simulation; 79: 145-159.

[16] Cheng, C., Chen, J., and Li, Z. (2010). A new algorithm for maximum likelihood estimation with progressive Type-I interval-censored data. Communications in Statistics-Simulation and Computation; 39: 750-766.

[17] Chen, D. G., and Lio, Y. L. (2010). Parameter estimations for generalized exponential distribution under progressive Type-I interval censoring. Computational Statistics and Data Analysis; 54: $1581-1591$.

[18] Lio, Y. L., Chen, D. G., and Tsai, T. R. (2011). Parameter estimations for generalized Rayleigh distribution under progressively Type-I interval-censored data. Open Journal of Statistics; 1: 46-57.

[19] Lin, Y. J., and Lio, Y. L. (2012). Bayesian inference under progressive type-I interval censoring. Journal of Applied Statistics; 39: 1811-1824.

[20] Teimouri, M., and Gupta, A. K. (2012). Estimation methods for the Gompertz-Makeham distribution under progressively Type-I interval censoring scheme. National Academy Science Letters; 35: 227-235.

[21] Singh, S., and Tripathi, Y. M. (2018). Estimating the Parameters of an inverse Weibull distribution under progressive type-I interval censoring. Statistical Papers; 59 (1): 21-56.

[22] Dempster, A. P., Laird, N. M., and Rubin, D. B. (1977). Maximum likelihood from incomplete data via the EM algorithm (with discussion). Journal of the Royal Statistical Society, B; 39: 1-39.

[23] Ihaka, R., and Gentleman, R. (1996). A language for data analysis and graphics. Journal of Computational and Graphical Statistics; 5: 299-314.

[24] Kemp, C. D., and Kemp, W. (1987). Rapid generation of frequency tables. Applied Statistics; 36: 277-282. 